\title{
ESTRÉS LABORAL FEMENINO Y POLÍTICAS DE IGUALDAD Y FLEXIBILIDAD EN ESPAÑA
}

\author{
FEMALE JOB STRESS AND WORK FAMILY AND EQUAL \\ POLICIES IN SPAIN
}

\author{
Consuelo LeÓn LlORENTE \\ Universidad Internacional de Cataluña
}

Recibido: 11/01/2016

Aceptado: 03/05/2016

\section{Resumen}

Este artículo recoge el marco legal, sociodemográfico y laboral de una etapa de gran crecimiento económico en España (2003-2007) que, sin embargo, resultó ser una oportunidad perdida en lo que respecta a la implantación efectiva de las políticas de conciliación e igualdad. En este periodo, la mujer española incrementó su tasa de incorporación al mundo laboral de un modo proporcionalmente mayor al resto de la población activa femenina europea. Sin embargo, la cultura hispana de largas jornadas laborales y el escaso liderazgo empresarial, resultaron ser un potente freno ante dos normas de hondo calado: la ley para la conciliación de la vida familiar y laboral (de 5 de noviembre, 39/1999) y la ley orgánica para la igualdad efectiva de mujeres y hombres (de 22 de marzo, LOIEMH, 3/2007). Diversas encuestas de salud pública y un estudio realizado a partir de una muestra de 4.700 empresas españolas, entre las que se encuentran 38 empresas del sector de la Comunicación y Publicidad, muestran un aspecto más en la tradicional brecha de género asociada a aspectos como salario, promoción y representación en puestos directivos. En este trabajo se pone de relieve los diferentes usos del tiempo, la doble jornada y su efecto en la correlación entre el estrés femenino, la fecundidad, el mercado laboral y la sostenibilidad social.

Palabras clave: conciliación trabajo-familia, horarios laborales, igualdad de género. 


\begin{abstract}
This article includes the legal, demographic and labour workforce in the context within a period of economic growth in Spain (2003-2007) which, however, turned out to be a missed opportunity as regards to the effective implementation of work-family and equal policies. In this period, the Spanish woman increases her rate of activity in the labour market in a greater proportion to the rest of the European labor workforce as women's work. However Hispanic culture of long working hours and poor corporate leadership, proved to be a powerful break before two deep draft standards: the Law for reconciling work and family life (of November 5, 39/1999) and Organic Law for the effective equality of women and men (of March 22, LOIEMH, 3/2007). Several public health surveys and a study from a sample of 4,700 Spanish companies, among them are 38 companies in the media and advertising sectors, show a new aspect of the traditional gender gap between men and women: salary, promotion, representation in management positions. This article discusses the different uses of time, women's dual role and the effects on the correlations between stress and fertility, labour market and social sustainability.
\end{abstract}

Keywords: work-family balance, work schedules, gender equality. 


\section{INTRODUCCIÓN}

Durante muchos años las empresas operaron sin preocuparse del impacto que su actividad fuera de control podía causar en el medio ambiente. Hoy existen normas y certificaciones de calidad que han cambiado los procedimientos y la mentalidad respecto a la responsabilidad no sólo social sino también ecológica -en su doble vertiente: medioambiental y social- de las empresas (Argandoña, Von Weltzien). Se abre paso, por tanto, un nuevo concepto, el de la «ecología humana», que vincula las acciones legales, empresariales y sociales de las compañías con la conducta, el perfil y los hábitos de vida de la población de un país (Diesendorf, Hamilton).

Contaminante es nuestro horario y contaminante es el estilo directivo de algunas empresas. En España vivimos inmersos en largas e improductivas jornadas laborales, con graves consecuencias para la salud -sindrome del burnout-; además de nuevas formas de discriminación, más sutiles que la diferencia salarial por género. Se trata de la discriminación por maternidad o paternidad actual o potencial ${ }^{1}$.

En este contexto, el denominado conflicto trabajo-familia está servido. Pero nuestro país no es el primero, aunque es cierto que en España se da de un modo peculiar. Básicamente esta situación es vivida como una disociación entre los dos roles: personal/profesional y laboral, en el contexto de unos horarios y una cultura de empresa que llevan a las personas a sentirlos como incompatibles (Greenhaus, Beutell).

La teoría de la ambivalencia instaura en la academia esta perspectiva dual a partir de los años 80. Especialmente desde la psicología social se incide en el estrés laboral de los padres y madres trabajadores (Lüscher, Pillemer).

Esta situación tiene efectos en la calidad de vida (Md-Sidin, Sambasivan, Ismail), la satisfacción vital (De Simone et al.) y el bienestar general, produciendo muchas veces estrés (Voydanoff). La lógica del mundo laboral -competitividad, prisas, eficiencia- parece haber invadido la esfera privada y

1. Ver el preámbulo de la Ley Orgánica 3/2007 para la igualdad efectiva de mujeres y hombres (LOIEMH). 
familiar imponiendo un estilo de vida poco sostenible desde la perspectiva de la «ecología social» que tiene sus ritmos y sus tiempos.

$\mathrm{Y}$ es que las empresas deberían contemplar su responsabilidad social también en su vertiente interna -respecto a sus empleados y empleadas- y no sólo en lo que respecta a sus acciones frente a proveedores y clientes (León 2010). Esta nueva actuación responsable configura sus verdaderos valores en acción, su misión como compañía respecto a la sociedad (Cardona, Rey).

La acción directiva y el consiguiente clima laboral es el contexto de algunas decisiones que se dan en el ámbito privado pero cuyas consecuencias tienen un enorme impacto social. Es el caso, por ejemplo, de las mujeres que deciden tener hijos o no tenerlos en función de las expectativas de contratación estable, promoción profesional y presencia o no de políticas denominadas «family friendly» en las compañías (Chinchilla, León 2004). De hecho en España, el retraso de la primera maternidad coincide con el asentamiento laboral de las mujeres que deciden o no ser madres en esos años (Delgado, Zamora; Miret, Cabré).

Este artículo recoge el marco legal, sociodemográfico y laboral de una etapa (2003-2007) caracterizada en España por una gran prosperidad y profusión legislativa pero a la vez, de escasa implantación efectiva de las políticas de conciliación, paridad y corresponsabilidad (Martín Llaguno, León Llorente, Guirao Mirón). La flexibilidad espacio temporal favorecida por el uso intensivo y generalizado de la tecnología no fue suficiente para cambiar prácticas y hábitos «tóxicos» especialmente en lo que se refiere al horario extenso y desordenado en nuestro país. España «no está en hora» y su desfase frente a Europa es en este punto un freno más de su productividad.

¿Qué importancia tuvieron la función directiva y la presión social del horario español en este periodo analizado? ¿Existió realmente una voluntad política de cambiar la situación a través de las dos leyes que regularon los nuevos derechos parentales del trabajador/a y que abundaban en las posibilidades de la flexibilidad y los permisos?

\section{CRECIMIENTO ECONÓMICO, AVANCE LEGISLACIÓN Y CULTURA DE EMPRESA}

La mujer en esta etapa incrementa su tasa de incorporación al mundo laboral de un modo proporcionalmente mayor al resto de Europa, situando a nuestro país cerca de los objetivos marcados por la estrategia de Lisboa: el 60\% en el año $2010^{2}$. La tasa de actividad femenina había pasado en España del 38,5\%

2. Ver en EUROSTAT.

Feminismo/s 27, junio 2016, pp. 243-261 
en 1996 al 52,65 \% en el 2010, mientras que la tasa de actividad masculina se mantenía estable desde el 66,1 \% en 1996 al 67,7 \% en el $2010^{3}$.

Se promulgaron además dos normas de hondo calado: la ley 39/1999, de 5 de noviembre, para promover la conciliación de la vida familiar y laboral y la ley Orgánica 3/2007, de 22 de marzo, para la igualdad efectiva de mujeres y hombres (LOIEMH), sin embargo la cultura de las empresas no cambió (Chinchilla, León 2011).

La primera de estas leyes tenía como objeto fomentar la incorporación de la mujer al mercado laboral en igualdad de condiciones que los varones. La creación de un Ministerio de Igualdad en el año 2008 hacía presuponer un cambio, sin embargo el gasto español favorable a estas políticas seguía siendo muy escaso. España junto con Italia son los países que menos gastan en familia ( $1,5 \%$ del PIB) en comparación con Finlandia o Suecia donde este porcentaje se sitúa en el 3\%.

Como contraste, en lo que respecta a las políticas activas de empleo, ocupamos una posición intermedia entre los países de la UE-15, con un gasto público que va del 0,63\% del PIB en el año 2007 (puesto 7/15) al 0,68\% del PIB en el 2010 (puesto 8/15). En otras palabras, la promoción de políticas de empleo sí fue una prioridad, tal como queda reflejado en el porcentaje que representan en el total del PIB destinado a estos programas, que nos sitúan por encima de la media de la UE-15 (Mínguez).

Si bien es cierto que en esta etapa -2003-2009- organismos públicos y privados pasaron de una primera etapa de sensibilización inicial a otra de implantación y difusión de políticas de igualdad y conciliación gracias a la promoción de los planes de igualdad y conciliación en las compañías, el porcentaje de empresas con una cultura flexible y conciliadora fue siempre muy bajo. Del 7\% inicial en el año 2003 (Poelmans, Chinchilla, Cardona) se llegó tan sólo al 12\% en el 20094 (León 2010).

Esta distancia entre el contexto legal y la cultura laboral en nuestro país es una de las cuestiones más debatidas en la opinión pública y a la vez menos estudiadas por la literatura académica (Martín Llaguno et al.). Además las mujeres durante la crisis económica en España asumieron en muchos casos el rol de principal mantenedor de una familia con todos los demás miembros en paro, siendo además sus contratos más precarios al estar tradicionalmente más afectados por la temporalidad y la desigualdad salarial. En esta etapa se da un claro descenso de la natalidad y un aumento de las tasas de estrés.

3. Ver en INE.

4. Ver en León 2010.

Feminismo/s 27, junio 2016, pp. 243-261 
En el mundo académico el tema de la igualdad, la conciliación, los horarios y la corresponsabilidad se abordó mayoritariamente desde estudios circunscritos al ámbito fundamentalmente intrafamiliar y femenino (Durán Heras). Son trabajos importantes, tanto por las conclusiones, como por la formulación de ciertas cuestiones. Muchos de ellos inciden en los sujetos pacientes de la conciliación: los hijos, una perspectiva que hoy, sin embargo, no parece estar tan presente.

Aunque con diferentes enfoques, todos coinciden en la importancia sociológica de las transformaciones y las nuevas dinámicas de las familias en las que ambos progenitores trabajan, todo ello potenciado por un uso intensivo de la tecnología, la organización flexible del trabajo y el desdibujamiento de los límites entre los dos ámbitos: trabajo y la familia. Las encuestas y estudios estadísticos oficiales reflejan estos enfoques pero analizan la situación como un problema limitado al ámbito privado y, por lo tanto, como una mera cuestión de uso y reparto del tiempo en el ámbito doméstico, de la distribución de roles y de tareas en el hogar (Meil).

Por el contrario en la UE, gracias a la legislación pionera en muchos países, al horario conciliador de todo el continente y a los programas EQUAL ${ }^{5}$, así como a los estudios sobre calidad de vida, empleo y sostenibilidad publicados por la European Foundation for the Improvement of Living and Working conditions $^{6}$, esta cuestión fue muy diferente. Desde finales de los años 70, las Encuestas de Condiciones de Trabajo y Salud (ECTS) ${ }^{7}$ se consolidaron como una eficaz herramienta de estudio acerca de las condiciones de trabajo y su impacto en la salud de la población trabajadora. Presentaban los factores de riesgo más comunes en los puestos de trabajo, las condiciones de contratación, la configuración del tiempo de trabajo, los recursos y acciones preventivas desarrolladas por las empresas, así como los daños a la salud derivados de las condiciones del entorno laboral.

\section{LA DOBLE JORNADA -TRABAJO Y FAMILIA- Y SU INCIDENCIA EN LA SALUD FEMENINA}

Familia y trabajo constituyen dos esferas esenciales en la percepción de la calidad de vida especialmente en las mujeres. La incorporación masiva de la mujer al mundo laboral y la normalización de las «parejas de doble ingreso»

5. Ver en The European Social Fund.

6. Ver en European Foundation for the Improvement of Living and Working conditions.

7. Ver en WHOQOL (The World Health Organization Quality of Life assessment). 
han implantado la perspectiva de «tensión entre roles», sobre todo en los países más desarrollados. En general, abunda el término conflicto, más que el de sinergia entre ambos mundos, haciendo hincapié en una visión clínica (efectos sobre la salud: estrés); sociológica (usos del tiempo) y más tarde centrada en la perspectiva de género ${ }^{8}$. Estos autores correlacionan el doble rol con una disminución de la calidad de vida y de satisfacción vital o bienestar.

Sin embargo, poco a poco se abre camino la teoría de la sinergia entre ambos mundos. En los años 90 surgen modelos teóricos que explican la relación positiva o beneficiosa entre trabajo y familia. Las competencias desarrolladas en un ámbito benefician al otro y viceversa. El empleado/a, que es a su vez ciudadano/a, cónyuge, padre o madre, lleva a todos esos ámbitos la satisfacción vital, calidad de vida y equilibrio lograda en su vida personal. Se habla de sinergias en vez de conflicto y por tanto de enriquecimiento entre estos dos mundos así como de complementariedad entre los diversos de roles del individuo?.

Según la Encuesta Mujer y Salud del 2008 solo 6 de cada 10 mujeres $(60,7 \%)$ percibían su estado de salud como bueno o muy bueno frente a un $72,7 \%$ de los hombres. En una encuesta anterior, en esta ocasión del Instituto de la Mujer del 2006 sobre usos del tiempo, esta diferencia resultaba estadísticamente significativa quedando corroborada la diferencia de género (Borrell, Artazcoz). La mujer española manifestaba un claro déficit especialmente en dos dominios: salud percibida y tiempo libre, por encima de otros temas como relación con la pareja, familia, vivienda, amistades, educación y situación económica (Requena, Suárez, Pérez).

En otra investigación realizada en torno a las demandas sociales más frecuentes en España respecto a los horarios, más del 50\% de los españoles consideraba que su trabajo no era flexible y en un $20 \%$ de los casos pensaba que tal como estaba organizado o del modo en que estaban distribuidas las cargas de trabajo, podían realmente realizarse en menos tiempo. El 70\% de los que

8. El término perspectiva de género aparece como tal en la literatura a partir del año 2000 incidiendo en la realidad de la división sexual del trabajo (Barnett, Hyde). En el modo de afrontar el conflicto, las mujeres adoptan una estrategia más reactiva que pro-activa (Beutell, Greenhaus 1982) y suelen tener escasa capacidad de negociación en el ámbito laboral (Matsui, Ohsawa,Onglatco) aunque su capacidad de atención múltiple para atender psicológicamente ambos mundos es mayor que la del varón. Lo desarrollan autores como Almeida, Wethington, Chandler; Frone, Russell, Cooper.

9. Las sinergias entre trabajo y familia fueron estudiadas fundamentalmente por Beutell, Greenhaus (1982, 1983); Frone, Russell, Cooper. 
afirmaban tener calidad de vida laboral, reconocían tener a la vez flexibilidad en la distribución de la jornada. Respecto a la salud, un 30\% afirmaba que no tener flexibilidad horaria en el puesto de trabajo les provocaba problemas de salud; si hablamos de personas en turnos de noche este porcentaje subía al 40\% (Sánchez 2012).

\section{POLÍTICAS DE FLEXIBILIDAD Y GESTIÓN DEL ESTRÉS EN LAS EMPRESAS}

Estas mismas cuestiones trasladadas al ámbito empresarial a través de una encuesta anual realizada por el IESE Business School, obtenían resultados muy similares. Estos resultados fueron sistematizados en una muestra de 4.700 empresas (2003-2007) por León $(2010)^{10}$. El cuestionario recogía una lista de treinta y una políticas y evaluaba aspectos facilitadores de la implantación de los programas tales como la sensibilización y ejemplaridad directiva respecto al plan de igualdad y conciliación, el presupuesto asignado, la comunicación interna de las medidas y el papel del equipo responsable encargado de llevarlo a cabo, normalmente mandos intermedios. La encuesta, por tanto, abordaba la disponibilidad y demanda de las políticas pero también la cultura empresarial que las respaldaba.

Como primer resultado destacable, cabe reseñar que la escasez de flexibilidad real y las largas jornadas laborales derivaron en absentismo y bajas por estrés, principales problemas en la empresa española durante este periodo.

Por otra parte y más allá de las frecuencias en el uso de las políticas, se constató que las correlaciones entre las distintas políticas entre sí eran más bajas que las correlaciones entre otros factores como el liderazgo y la cultura de empresa (ver tabla 1 y gráfico 1). Esto significa que en los programas de conciliación y de igualdad, la actitud directiva ante estas cuestiones influía en la cultura final de la compañía, mucho más que el diseño concreto de un determinado programa. La confianza del directivo ante el uso que hacía el empleado/a de estas medidas, actuaba como impulsor o por el contrario como freno de los programas.

10. Este trabajo -basado en los datos recogidos en el índice IFREI (Family Responsible Employer Index) del IESE Business School durante este periodo- fueron objeto de la tesis doctoral de Consuelo León. 
Tabla 1: Políticas de flexibilidad en 4.700 empresas españolas (2003-2007)

\begin{tabular}{|c|c|c|c|c|c|c|c|c|c|c|c|}
\hline & $\begin{array}{l}\text { Horario } \\
\text { flexible }\end{array}$ & $\begin{array}{l}\text { Tiempo } \\
\text { parcial }\end{array}$ & $\begin{array}{c}\text { Semana } \\
\text { comprimida }\end{array}$ & $\begin{array}{l}\text { Jornada } \\
\text { reducida }\end{array}$ & Exced & $\begin{array}{l}\text { Vac. } \\
\text { Flex. }\end{array}$ & $\begin{array}{l}\text { Tele- } \\
\text { trabajo }\end{array}$ & $\begin{array}{l}\text { Ase. } \\
\text { Prof. }\end{array}$ & $\begin{array}{l}\text { Ases. } \\
\text { Pers. }\end{array}$ & $\begin{array}{l}\text { Form. } \\
\text { Con. }\end{array}$ & $\begin{array}{l}\text { Form. } \\
\text { Tiem. }\end{array}$ \\
\hline Tiempo parcial & 0,27 & & & & & & & & & & \\
\hline $\begin{array}{l}\text { Semana } \\
\text { comprimida }\end{array}$ & 0,24 & 0,27 & & & & & & & & & \\
\hline $\begin{array}{l}\text { Jornada } \\
\text { reducida }\end{array}$ & 0,24 & 0,43 & 0,19 & & & & & & & & \\
\hline Excedencia & 0,14 & 0,20 & 0,17 & 0,20 & & & & & & & \\
\hline $\begin{array}{l}\text { Vacaciones. } \\
\text { Flexibles }\end{array}$ & 0,07 & 0,04 & 0,10 & 0,04 & 0,25 & & & & & & \\
\hline Teletrabajo & 0,35 & 0,22 & 0,19 & 0,20 & 0,13 & 0,07 & & & & & \\
\hline $\begin{array}{l}\text { Asesoramiento } \\
\text { Profesional }\end{array}$ & 0,12 & 0,14 & 0,11 & 0,13 & 0,16 & 0,10 & 0,17 & & & & \\
\hline $\begin{array}{l}\text { Asesoramiento } \\
\text { Personal }\end{array}$ & 0,12 & 0,13 & 0,09 & 0,12 & 0,13 & 0,03 & 0,14 & 0,42 & & & \\
\hline Form.Concilia. & 0,14 & 0,12 & 0,09 & 0,09 & 0,13 & 0,04 & 0,15 & 0,22 & 0,29 & & \\
\hline $\begin{array}{l}\text { Form.Gest. } \\
\text { Tiempo }\end{array}$ & 0,08 & 0,08 & 0,09 & 0,06 & 0,14 & 0,12 & 0,08 & 0,21 & 0,18 & 0,28 & \\
\hline Guardería & 0,14 & 0,15 & 0,13 & 0,11 & 0,13 & 0,04 & 0,18 & 0,14 & 0,21 & 0,21 & 0,13 \\
\hline
\end{tabular}

La cultura de empresa incluía también cuestiones como la no existencia de represalias en la promoción y en la trayectoria profesional debido al uso por parte del empleado de unas políticas que en realidad estaban respaldas por la ley. La implementación de políticas requería, por tanto, una cultura «family friendly», flexible, familiarmente responsable que de modo formal e informal animara los programas. No bastaba la presión institucional, era preciso que el programa fuera realidad en la cultura de las empresas (Cross).

El temor de los directivos a la flexibilidad respondía y de hecho responde también hoy, al desconocimiento de las herramientas de dirección para organizar el trabajo de otra manera. Cuando la flexibilidad espacio-temporal no es resultado de un cambio profundo en los procedimientos internos de la compañía; algunas prácticas como el teletrabajo o la flexibilidad horaria pueden derivar en una cultura tóxica cuyo efecto más inmediato es el estrés (León 2015).

Una muestra más del desconocimiento que existe de la verdadera naturaleza del problema es que según este mismo estudio, en esos años, la demanda de cursos en formación en gestión del tiempo y del estrés creció, no así los programas de formación directiva en gestión de la diversidad o conciliación trabajo y familia. Se atacaba de nuevo el efecto y no la causa del problema. 
Gráfico 1: Políticas y prácticas más frecuentemente asociadas en 4.700 empresas españolas (2003-2007)

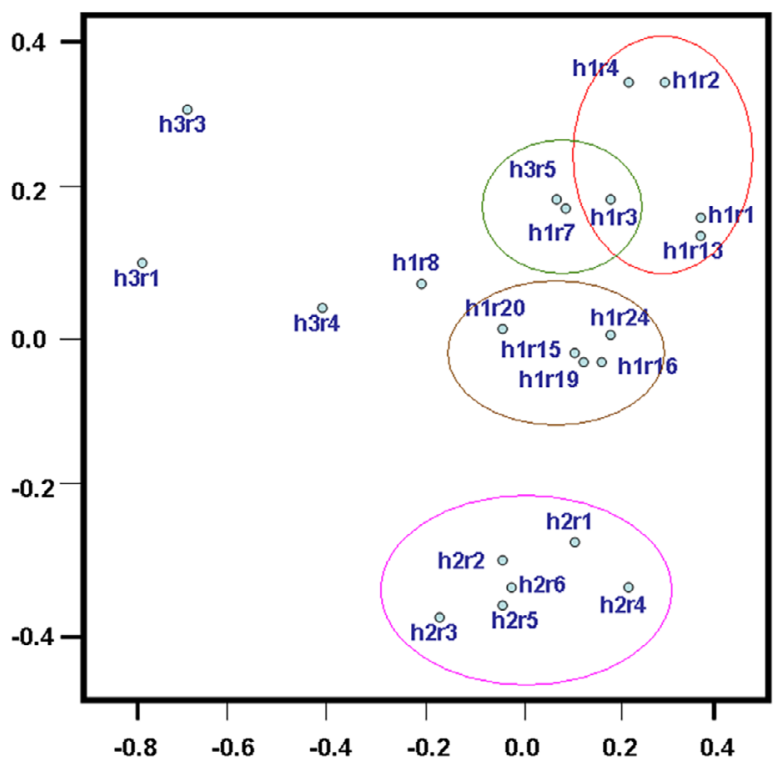

Bloque de políticas

H1R1: Horario flexible

H1R2: Trabajo a tiempo parcial

H1R3: Medio día libre a cambio de alargar la jornada

H1R4: Jornada reducida

H1R7: Excedencia para cuidar de un familiar

H1R8: Vacaciones flexibles

H1R13: Trabajar desde casa

H1R15: Asesoramiento profesional

H1R16: Asesoramiento personal/familiar

H1R19: Formación sobre conciliar trabajo y familia

H1R20: Formación en gestión del tiempo y estrés

H1R24: Servicio o subvención de guardería

\section{Cultura directiva}

H2R1: Acorde con la conciliación y equidad en su discurso verbal

H2R2: Acorde con la conciliación y equidad en su horario personal

H2R3: Se ha diseñado un programa de conciliación y equidad

H2R4: Se ha asignado un presupuesto al programa

H2R5: Se ha comunicado internamente el programa

H2R6: Se ha asignado un responsable a la implantación del programa

\section{Cultura empresarial}

H3R1: Llevarse trabajo a casa es una práctica habitual

H3R3: Quien hace uso de las medidas de conciliación muestra escaso compromiso H3R4: No es frecuente quedarse en la oficina más allá del horario laboral

H3R5: La situación familiar es una variable presente en el Departamento de RRHH 


\section{PROBLEMAS MÁS RELEVANTES EN LAS EMPRESAS DE MEDIOS DE COMUNICACIÓN Y PUBLICIDAD}

Cuando se analiza con más en detalle cuáles eran los problemas más relevantes de la empresa española en ese periodo, los directivos encuestados señalaron fundamentalmente dos: la dificultad para encontrar empleados clave y el absentismo (ver Total Sectores en Tabla 2).

Tabla 2: Principales problemas de las empresas españolas en relación al sector de actividad

\begin{tabular}{|c|c|c|}
\hline Sector & Primer problema & Segundo problema \\
\hline Telecomunicaciones & $\begin{array}{l}\text { Empleados clave } \\
(38 \%)\end{array}$ & $\begin{array}{l}\text { Poca iniciativa } \\
(30 \%)\end{array}$ \\
\hline Distribución y Logística/Transporte & $\begin{array}{l}\text { Empleados clave } \\
(60 \%)\end{array}$ & $\begin{array}{l}\text { Poca iniciativa } \\
(43 \%)\end{array}$ \\
\hline Hotelería y Restauración/Ocio & Absentismo (38\%) & $\begin{array}{l}\text { Conciliar trabajo y } \\
\text { familia }(38 \%)\end{array}$ \\
\hline Publicidad y medios de comunicación & $\begin{array}{l}\text { Empleados clave } \\
(59 \%)\end{array}$ & Absentismo (28\%) \\
\hline Banca, finanzas y seguros/Salud & $\begin{array}{l}\text { Resistencia a la } \\
\text { movilidad (40\%) }\end{array}$ & $\begin{array}{l}\text { Conciliar trabajo y } \\
\text { familia }(39 \%)\end{array}$ \\
\hline $\begin{array}{l}\text { Producción o distribución de energía o } \\
\text { agua/Química, petróleo, gas, caucho y } \\
\text { plásticos }\end{array}$ & Absentismo (47\%) & $\begin{array}{l}\text { Poca iniciativa } \\
(39 \%)\end{array}$ \\
\hline Metalúrgico & Absentismo (60\%) & $\begin{array}{l}\text { Empleados clave } \\
(48 \%)\end{array}$ \\
\hline $\begin{array}{l}\text { Industria electrónica/ Industria del } \\
\text { software }\end{array}$ & $\begin{array}{l}\text { Resistencia a la } \\
\text { movilidad ( } 46 \%)\end{array}$ & $\begin{array}{l}\text { Poca iniciativa } \\
(38 \%)\end{array}$ \\
\hline Industria de automoción & Absentismo (54\%) & $\begin{array}{l}\text { Empleados clave } \\
(38 \%)\end{array}$ \\
\hline Alimentos, bebidas y tabaco & $\begin{array}{l}\text { Resistencia a la } \\
\text { movilidad (50\%) }\end{array}$ & Absentismo (50\%) \\
\hline $\begin{array}{l}\text { Textil, calzado, confección, cuero/ } \\
\text { Madera, papel, artes gráficas }\end{array}$ & Absentismo (70\%) & $\begin{array}{l}\text { Empleados clave } \\
(46 \%)\end{array}$ \\
\hline Construcción & $\begin{array}{l}\text { Empleados clave } \\
(54 \%)\end{array}$ & $\begin{array}{l}\text { Conciliar trabajo y } \\
\text { familia (33\%) }\end{array}$ \\
\hline Total Sectores & $\begin{array}{l}\text { Empleados clave } \\
(48 \%)\end{array}$ & Absentismo (35\%) \\
\hline
\end{tabular}

Esta situación se hacía más crítica en las 38 empresas del sector de Publicidad y Medios de Comunicación analizadas. Fuertemente feminizado y precario en 
sus condiciones laborales - altas tasas de temporalidad- en este sector la flexibilidad no ha sido un logro sino más bien una realidad peculiar -disponibilidad a todas horas- que el uso intensivo de la tecnología agudizó (León 2015).

Demandas a horas intempestivas en el contexto de un mundo global e interconectado permanentemente hacen de este ámbito, la Publicidad y los Medios de Comunicación, un subgrupo profesional en el que puede afirmarse que la dos leyes marco de esta década- la referente a la conciliación de la vida familiar y laboral (de 5 de noviembre, 39/1999) y la ley orgánica para la igualdad efectiva de mujeres y hombres (de 22 de marzo, LOIEMH, 3/2007)actuaron como un verdadero boomerang para las mujeres ${ }^{11}$.

Como resultado, ellas seguían ganando menos, no tenían los hijos que deseaban (Escobedo, Navarro, Flaquer) y trabajaban de forma más precaria en una situación clara de doble jornada. En aquellas empresas con mayor porcentaje de mujeres en plantilla -más del 75\%- el estrés y el absentismo laboral eran los primeros problemas señalados por los directivos encuestados (ver tabla 3).

Paradójicamente esta situación - ausencia de programas de igualdad y conciliación- no revertía en un descenso del compromiso aunque sí estaba correlacionado con el crecimiento del absentismo, muchas veces estrés encubierto de mujeres que no podían conciliar.

Tabla 3: Principales problemas de las empresas españolas en relación al porcentaje de mujeres en plantilla

\begin{tabular}{|l|c|c|c|c|}
\hline & \multicolumn{3}{|c|}{$\%$ de mujeres en la plantilla } \\
\cline { 2 - 5 } & $\begin{array}{c}\text { Menos } \\
\text { del 25\% }\end{array}$ & $\begin{array}{c}\text { Del 25 } \\
\text { al 50\% }\end{array}$ & $\begin{array}{c}\text { Del 50 } \\
\text { al 75\% }\end{array}$ & $\begin{array}{c}\text { Más del } \\
75 \%\end{array}$ \\
\hline $\begin{array}{l}\text { Resistencia de los empleados a la movilidad } \\
\text { geográfica }\end{array}$ & & $\mathrm{x}$ & & \\
\hline Absentismo & & & & $\mathrm{x}$ \\
\hline Dificultad para contratar empleados clave & $\mathrm{x}$ & & & \\
\hline Dificultad para conciliar trabajo y familia & & & $\mathrm{x}$ & \\
\hline Bajas por estrés & & & $\mathrm{x}$ & $\mathrm{x}$ \\
\hline $\begin{array}{l}\text { Falta de compromiso por parte de los } \\
\text { empleados }\end{array}$ & $\mathrm{x}$ & & & \\
\hline Poca iniciativa por parte de los empleados & & $\mathrm{x}$ & $\mathrm{x}$ & \\
\hline
\end{tabular}

11. Esta situación fue avanzada ya por Smart en 1989 en su conocida obra Feminism and the Power of Law. 
Cuando además en estas empresas se daban altos índices de temporalidad y precariedad; la dificultad para conciliar trabajo y familia, la ausencia de compromiso y las dificultades para retener talento aparecen en primer lugar (ver tabla 4).

Tabla 4: Principales problemas de las empresas españolas en relación al porcentaje de empleados con contrato temporal

\begin{tabular}{|c|c|c|c|}
\hline & \multicolumn{3}{|c|}{$\begin{array}{c}\% \text { de empleados con contratos } \\
\text { temporales }\end{array}$} \\
\hline & $\begin{array}{l}\text { Menos del } \\
10 \%\end{array}$ & $\begin{array}{l}\text { Del } 10 \text { al } \\
20 \%\end{array}$ & $\begin{array}{c}\text { Más del } \\
20 \%\end{array}$ \\
\hline $\begin{array}{l}\text { Resistencia de los empleados a la movilidad } \\
\text { geográfica }\end{array}$ & $\mathrm{x}$ & & \\
\hline Absentismo & & $\mathrm{x}$ & \\
\hline Dificultad para contratar empleados clave & & & $\mathrm{x}$ \\
\hline Dificultad para conciliar trabajo y familia & & & $\mathrm{x}$ \\
\hline Bajas por estrés & & $\mathrm{x}$ & \\
\hline $\begin{array}{l}\text { Falta de compromiso por parte de los } \\
\text { empleados }\end{array}$ & & & $\mathrm{x}$ \\
\hline Poca iniciativa por parte de los empleados & & $\mathrm{x}$ & \\
\hline
\end{tabular}

\section{RESPONSABILIDAD SOCIAL DE LA EMPRESA Y SUS EFECTOS EN LA SALUD}

En España, las formas de organización del trabajo y los estilos directivos necesitan abordar una profunda transformación, si las empresas no quieren dejar de ser competitivas y globales frente a sus colegas de otros países. Es preciso cumplir plenamente la legislación, pero eso no es suficiente. Se trata de apostar por un pacto social que armonice horarios laborales, escolares, comerciales y familiares, para atacar de raíz los males de nuestra cultura laboral, nuestro modo de trabajar: el horario.

La Plataforma de la Racionalización de Horarios $\mathrm{ARHOE}^{12}$ ha propuesto algunas medidas: impulsar el horario europeo, con objeto de armonizar comercialmente España con el resto de países; favorecer las jornadas continuadas favoreciendo así una gestión productiva del tiempo personal; sincronizar la jornada laboral de los padres con la jornada escolar de sus hijos; adelantar el

12. Ver Asociación para la Comisión Nacional para la Racionalización de los Horarios Españoles (ARHOE) 
horario de los restaurantes y de la programación televisiva, especialmente de los espacios de informativos; impulsar los servicios on line desde la administración pública, los bancos y los comercios; integrar en el sistema educativo el verdadero sentido de la corresponsabilidad varón-mujer en los distintos ámbitos, una cuestión que va más allá del reparto de tareas asociadas a un rol.

Esta postura ha sido también suscrita por la International Labour Organization (ILO) que hizo recomendaciones muy específicas a las empresas animando a la adopción de programas trabajo-familia WLB (Work Life Balance) como un paso más en las políticas de igualdad. En ocasiones, estos programas centrados excesivamente en la cuestión de las cuotas, la igualdad salarial o la promoción no son tan eficaces en las propuestas y en la resolución cotidiana de la corresponsabilidad en el hogar. La integración plena de la mujer en el mundo laboral pasa por la normalización de la maternidad como hecho social hasta convertirla en objeto de especial protección por los poderes públicos, máxime en un contexto de invierno demográfico ya mencionado y de insostenibilidad de nuestro estado del bienestar (León Borja).

En países como Holanda, Suecia, Noruega, Francia y Alemania, la racionalización de horarios ha hecho posible tres objetivos simultáneos: mayor incorporación de la mujer al mundo laboral, aumento del índice de natalidad y mayor productividad en relación al número de horas trabajadas por empleado.

La empresa, con políticas de empleo flexibles y con una cultura horaria razonable y productiva puede contribuir y mucho al desarrollo sostenible y a la salud de la sociedad. Sus políticas, por tanto, no son sólo políticas de empleo sino también políticas sociales.

Por este motivo, las medidas de conciliación y flexibilidad, como conjunto de prácticas formalizadas que apoyan la integración entre trabajo y vida personal, son capaces también de fomentar la equidad en el cumplimiento de responsabilidades entre hombres y mujeres, e influyen también en la corresponsabilidad intrafamiliar (O'Driscoll et al.).

En general, las políticas de flexibilidad, los beneficios extra salariales y los servicios tienen un elevado impacto en las vidas de los/as empleados/as, pues facilitan a las personas organizar mejor su tiempo y favorecen su rendimiento, disminuyendo además la rotación de los empleados clave y atrayendo talento, especialmente femenino. Las mujeres habitualmente anteponen flexibilidad espacio temporal a salario. En estas empresas se produce una mejora de las bajas por estrés y del absentismo que muchas encubre necesidades de 
conciliación ${ }^{13}$. Mejora también la innovación hasta el punto de que puede hablarse de un retorno de la inversión realizada en estos programas (Balmforth, Gardner).

Respecto al capital social del futuro -los jóvenes- la temporalidad en el empleo ha generado además nuevas situaciones en lo que respecta a la cuestión de la conciliación trabajo y vida personal/familiar. La separación de la pareja o de los cónyuges que viven y trabajan en ciudades o países diferentes ya no es una excepción. Nuestros jóvenes viven de un modo global, cambiante y precario y podemos afirmar que los primeros años de la actividad laboral, después de haber realizado sus estudios universitarios, se han convertido en una carrera de obstáculos en la que a la batalla por la seguridad se une la incertidumbre en su vida personal/familiar.

En épocas de escasez de talento -esta situación se producirá cuando se jubilen o fallezcan los «baby boomers»- las empresas deberán plantearse en serio cómo ser atractivas para los perfiles que buscan. Las nuevas generaciones incorporan cada vez más la calidad de vida entre los criterios por los que deciden dónde quieren trabajar, situando esta aspiración en el mismo nivel que la cuestión salarial. La familia del empleado, sus intereses, su proyecto personal, serán, por tanto, cada vez más, verdaderos «stakeholders» de la empresa, agentes de interés cuyo impacto interno podrá compararse al de accionistas, proveedores y clientes.

Puede afirmarse, por tanto, que existe un «lucro cesante», un beneficio que se deja de percibir cuando no existen estas políticas. A nivel internacional, diversos estudios hablan ya de este coste de oportunidad debido a los efectos indirectos de una deficiente organización de la vida laboral, poco flexible, no acorde con la globalización de los mercados y a las nuevas demandas horarias de una sociedad en la que ambos progenitores trabajen. Las empresas mecanicistas, rígidas en horarios, y poco sensibles al entorno personal y familiar del empleado serán menos atractivas y perderán no sólo capital humano sino que serán «contaminantes» y poco sostenibles con el entorno económico, social y demográfico (Abbott, De Cieri). Renunciar a estar en vanguardia en estas cuestiones es un precio demasiado alto a pagar. En esta ocasión, una vez más, lo verdaderamente rentable es tener muy en cuenta el largo plazo.

13. En la tesis de Consuelo León realizada en el año 2010 se confirman trabajos anteriores realizados en diferentes papers de referencia en el tema. Ver Grover, Crooker; Ingram, Simons; Kossek,Ozeki. 


\section{AGRADECIMIENTOS}

Este trabajo se enmarca en el ámbito del Grupo de Investigación Parentalidad, Igualdad y Conciliación (PIC), reconocido como Grupo Emergente por la Generalitat de Catalunya (2014 SGR 1229) y coordinado desde el Instituto de Estudios Superiores de la Familia de la Universitat Internacional de Catalunya. El Grupo forma parte de la Cátedra IsFamily Santander (Intergenerational Solidarity in the Family).

\section{REFERENCIAS BIBLIOGRÁFICAS}

Abbott, Jacqui y Helen De Cieri. «Influences on the provision of Work-life benefits: Management and employee perspectives». Journal of Management and Organization 14.3 (2008): 303-320.

Almeida, David M., Elaine Wethington y Amy L. Chandler. «Daily transmission of tensions between marital dyads and parent-child dyads». Journal of Marriage and the Family (1999): 49-61.

Argandoña, Antonio y Heidi von Weltzien Hoivik. «Corporate social responsibility: One size does not fit all. Collecting evidence from Europe». Journal of Business Ethics 89.3 (2009): 221-234.

ARHOE (Asociación para la Comisión Nacional para la Racionalización de los Horarios Españoles). <http://www.horariosenespana.com/images/manifiesto. pdf>, consultado el 29-04-2016.

Balmforth, Kelly y Dianne Gardner. «Conflict and facilitation between work and family: Realizing the outcomes for organizations». New Zealand Journal of Psychology 35.2 (2006): 69-76.

Barnett, Rosalind Chait y Janet Shibley Hyde. «Women, men, work, and family». American psychologist 56.10 (2001): 781-787.

Beutell, Nicholas J. y Jeffrey H. Greenhaus. «Interrole conflict among married women: The influence of husband and wife characteristics on conflict and coping behavior». Journal of Vocational Behavior 21.1 (1982): 99-110.

Beutell, Nicholas J. y Jeffrey H. Greenhaus. «Integration of home and nonhome roles: Women's conflict and coping behavior». Journal of Applied Psychology 68.1 (1983): 43-50.

Borrell, Carme y Lucía Artazcoz. «Las desigualdades de género en salud: retos para el futuro». Revista Española de Salud Pública 82.3 (2008): 241-249.

Cardona, Pablo y Carlos Rey. Dirección por misiones: primeras experiencias de éxito. Economistas 24.109 (2006): 101-107.

Chinchilla, Nuria y Consuelo León. La ambición femenina. Cómo reconciliar familia y trabajo. Madrid: Aguilar, 2004. 
Chinchilla, Nuria y Consuelo León. Diez años de conciliación en España. Madrid: Grupo 5, 2011.

Cross, Christine. «Barriers to the executive suite: evidence from Ireland». Leadership \& Organization Development Journal 31.2 (2010): 104-119.

De Simone, Silvia, Jessica Lampis, Diego Lasio, Francesco Serri, Gianfranco Cicotto y Daniela Putzu. «Influences of work-family interface on job and life satisfaction». Applied Research in Quality of Life 9.4 (2014): 831-861.

Delgado, Margarita y Francisco Zamora. «Españolas y extranjeras: su aportación a la fecundidad en España». Economistas 22.99 (2004): 88-97.

Diesendorf, Mark y Clive Hamilton. Human Ecology, Human Economy: Ideas for an ecologically sustainable future. St. Leonard: NSW: Allen \& Unwin, 1997.

Durán Heras, María Ángeles. «La investigación sobre el uso del tiempo en España: algunas reflexiones metodológicas». Revista Internacional de Sociología 18 (1997):163-193.

Escobedo, Anna, Lara Navarro y Luis Flaquer. Perspectivas de desarrollo y evaluación de las políticas de licencias parentales y por motivos familiares en España y en la Unión Europa FIPROS. Madrid: Ministerio de Trabajo, 2007.

European Foundation for the Improvement of Living and Working conditions. <http://www.eurofound.europa.eu/>, consultado el 16-04-2016.

EUROSTAT. Eurostat Regional Yearbook 2010. <http://ec.europa.eu/eurostat/web/ products-statistical-books/-/KS-HA-10-001>, consultado el 16-04-2016.

Frone, Michael R., Marcia Russell y M. Lynne Cooper. «Relation of work-family conflict to health outcomes: A four-year longitudinal study of employed parents». Journal of Occupational and Organizational psychology 70.4 (1997): 325-335.

Greenhaus, Jeffrey H. y Nicholas J. Beutell. «Sources of conflict between work and family roles». Academy of management review 10.1 (1985): 76-88.

Grover, Steven L. y Karen J. Crooker. «Who appreciates family-responsive human resource policies: The impact of family-friendly policies on the organizational attachment of parents and non-parents». Personnel psychology 48.2 (1995): 271-288.

ILO (International Labour Organization). World Employment and Social Outlook: Trends 2016. < http://www.ilo.org/global/research/global-reports/weso/2016/ WCMS_443480/lang-en/index.htm>, consultado el 16-04-2016.

INE (Instituto Nacional de Estadística). Encuesta Población Activa 2010. <http:// www.ine.es/prensa/epa_tabla.htm>, consultado el 16-04-2016.

Ingram, Paul y Tal Simons. «Institutional and resource dependence determinants of responsiveness to work-family issues». Academy of Management Journal 38.5 (1995): 1466-1482. 
Kossek, Ellen Ernst y Cynthia Ozeki. «Bridging the work-family policy and productivity gap: A literature review». Community, Work \& Family 2.1 (1999): 7-32.

León Borja, Margarita (Coord.). Empleo y maternidad: obstáculos y desafíos a la conciliación de la vida laboral y familiar. Barcelona: IGOP-FUNCAS, 2015.

León, Consuelo. Factores de éxito en la implantación de políticas trabajo y familia en las empresas españolas (2003-2007) (Tesis Doctoral). Barcelona: Universitat Politécnica de Catalunya, 2010.

León, Consuelo «¿Flexitime» versus estrés?». Harvard Deusto Business Review 243 (2015): 24-33.

Ley Orgánica 3/2007 para la igualdad efectiva de mujeres y hombres (LOIEMH).

<https://www.boe.es/buscar/doc.php?id=BOE-A-2007-6115>, consultado el 16-04-2016.

Lüscher, Kurt y Karl Pillemer. «Intergenerational ambivalence: A new approach to the study of parent-child relations in later life». Journal of Marriage and the Family 60 (1998): 413-425.

Martín Llaguno, Marta, Consuelo León Llorente y Cristina Guirao Mirón. «La conciliación familiar y laboral en España en la esfera política, mediática y empresarial. Un estudio de agendas entre 2003 y 2007». Cuestiones de género: de la igualdad y la diferencia 8 (2013): 145-164.

Matsui, Tamao, Takeshi Ohsawa y Mary-Lou Onglatco. «Work-family conflict and the stress-buffering effects of husband support and coping behavior among Japanese married working women». Journal of Vocational Behavior 47.2 (1995): 178-192.

Md-Sidin, Samsinar, Murali Sambasivan e Izhairi Ismail. «Relationship between work-family conflict and quality of life: An investigation into the role of social support». Journal of Managerial Psychology 25.1 (2010): 58-81.

Meil, Gerardo. «El papel de los niños en la redefinición del trabajo doméstico en la nueva familia urbana española». Revista Internacional de Sociología 16 (1997): 39-56.

Miret, Pau y Anna Cabré Pla. «Pautas recientes en la formación familiar en España: Constitución de la pareja y fecundidad». Papeles de Economía Española 104 (2005): 17-36.

Moreno Mínguez, Almudena. «La protección familiar en España: un reto aplazado». Presupuesto y gasto público 71 (2013): 223-239.

O'Driscoll, Michael, Steven Poelmans, Paul Spector, Thomas Kalliath, Tammy Allen...Juan I. Sanchez. «Family-responsive interventions, perceived organizational and supervisor support, work-family conflict, and psychological strain». International Journal of Stress Management 10.4 (2003): 326-332.

Sánchez Laguna, Pilar. Demandas sociales en materia de horarios. Madrid: Editorial Dykinson, 2012. 
Poelmans, Steven, Nuria Chinchilla y Pablo Cardona «The adoption of familyfriendly HRM policies: Competing for scarce resources in the labour market». International Journal of Manpower 24.2 (2003): 128-147.

Requena, María Luisa, Mónica Suárez y Óscar Pérez. «Encuestas de salud en España: situación actual». Revista Española de Salud Pública 87.6 (2013): 549-573.

Smart, Carol. Feminism and the Power of Law. London: Editorial Routledge, 1989.

The European Social Fund. <http://www.esf.ie/en/Programmes/Other-ESF-Programmes-2000-2006/EQUAL-Operational-Programme-2000-2006>, consultado el 16-04-2016.

Voydanoff, Patricia. «Consequences of boundary-spanning demands and resources for work-to-family conflict and perceived stress». Journal of occupational health psychology 10.4 (2005): 491-496.

WHOQOL (The World Health Organization Quality of Life assessment).

<http://www.who.int/mental_health/publications/whoqol/en/>, consultado el 16-04-2016. 